Relations industrielles

Industrial Relations

\title{
Emploi et conditions de travail du personnel infirmier, Genève, Bureau International du Travail, Série Etudes et Documents, nouvelle série No 55. 1960, 198 pp.
}

\section{Jacques Archambault}

Volume 15, numéro 3, juillet 1960

URI : https://id.erudit.org/iderudit/1021967ar

DOI : https://doi.org/10.7202/1021967ar

Aller au sommaire du numéro

Éditeur(s)

Département des relations industrielles de l’Université Laval

ISSN

0034-379X (imprimé)

1703-8138 (numérique)

Découvrir la revue

Citer ce compte rendu

Archambault, J. (1960). Compte rendu de [Emploi et conditions de travail du personnel infirmier, Genève, Bureau International du Travail, Série Etudes et Documents, nouvelle série No 55. 1960, 198 pp.] Relations industrielles /

Industrial Relations, 15(3), 384-385. https://doi.org/10.7202/1021967ar

Tous droits réservés (C Département des relations industrielles de l’Université Laval, 1960
Ce document est protégé par la loi sur le droit d'auteur. L'utilisation des services d’Érudit (y compris la reproduction) est assujettie à sa politique d'utilisation que vous pouvez consulter en ligne.

https://apropos.erudit.org/fr/usagers/politique-dutilisation/ 
l'homme comme un pur objet d'études ou d'expérimentation. Seules, une juste conception de l'homme permettra de les employer comme il convient pour le libérer de ses déterminismes par la connaissance de soi-même et par l'éducation.

Un mouvement comme celui des relations humaines, qui découle des sciences de l'homme et met en oeuvre des techniques d'action sur l'homme, peut montrer, dans les circonstances actuelles, une véritable utilité, à la condition qu'on ne le réduise point à une série de procédés ou de recettes, mais qu'il soit la mise en oeuvre, dans tous les domaines, d'un esprit caractérisé par le respect de la personne et la volonté de lui assurer une participation à l'activité des groupes.

De même, le progrès des conceptions et des techniques juridiques qui, depuis la prise de conscience temporelle des droits de l'homme, a déjà beaucoup servi à la personne, peut continuer à la protéger et à la favoriser par l'entrée en Droit positif des attributs de la personne humaine comme par un effort pour la restauration des initiatives et de la responsabilité de chaque personne.

\section{RECENSIONS - BOOK REVIEWS}

Emploi et conditions de travail du personnel infirmier, Genève, Bureau International du Travail, Série Etudes et Documents, nouvelle série No 55. $1960,198 \mathrm{pp}$.

On connait l'intérêt et la qualité des études du B.I.T. Cette étude qui vient de nous arriver est à la fois le rapport d'une enquête effectuée dans 56 pays ou territoires et un compterendu des travaux d'une réunion spéciale sur les conditions de travail et d'emploi du personnel infirmier tenue à Genève du 6 au 11 octobre 1958. Des experts de 14 pays ont participé à la réunion, dont le Canada, représenté par le Secrétaire du Service des Infirmières de l'Association canadienne du personnel infirmier, (Ottawa) Mlle Lillian Campion. Il faut noter d'abord, que cette étude ne s'applique qu'aux infirmières ou infirmiers proprement dits et au personnel auxiliaire exécutant, dans les hôpitaux ou dans les services publics ou privés, des tâches étroitement connexes à celle d'infirmières.

Le premier chapître traite, d'une facon fort générale, des fonctions et qualifications du personnel infirmier. Le chapître II aborde les problèmes de l'emploi. La conclusion est nette «à l'heure actuelle, la situation de l'emploi du personnel infirmier se caractérise surtout par l'insuffisance de l'effectif du personnel qualifié par rapport aux besoins \& présents et futurs ». Presque partout dans le monde, l'offre de personnel infirmier est de beaucoup inférieure à la demande».

Dans «l'étude des mesures visant à éliminer la pénurie du personnel » (p. 40 et suivantes) on en vient facilement et premièrement au fait que \&les conditions de travail et le statut du personnel infirmier soient de nature à attirer et retenir des candidats capables et soutiennent la comparaison avec les conditions qui règnent dans d'autres professions et carrières comparables ». En effet, \&il semble, toutefois, ressortir de la documentation existante, que le taux élevé des défections et l'échec des systèmes de recrutement s'expliquent, dans une très large mesure, par l'état peu satisfaisant des conditions de vie et de travail et par la situation sociale des infirmières qui sont sans commune mesure avec leur degré d'instruction, leurs fonctions et leurs responsabilités » (p. 55)

Les chapitres III à VIII inclusivement traitent des conditions de travail: 
1-De la façon avec laquelle elles sont déterminées; 41 sur 54 le sont par procédure de négociations. 2-Durée du travail, congés, repos hebdomadaires, vacances. 3-Rémunération. 4-Conditions de vie. 5-Protection de la santé. 6-Sécurité sociale.

Les données quant à la rémunération ne nous permettent pas d'établir des comparaisons d'un pays ou d'une région à l'autre «il semble évident, toutefois, que dans de nombreux pays, la rémunération des infirmières soit fixée sans qu'on tienne grand compte de la formation, du travail, des fonctions et des responsabilités des intéressés 》 (p. 110)

On signale, qu'à l'heure actuelle, le principal problème semble être celui de la durée du travail: longues heures, heures brisées, travail du dimanche, travaii de nuit, travail de fins de semaines etc. etc.

Enfin, l'étude citée reproduit, aux pages 188 à 195, les « recommandations de la réunion spéciale sur les conditions de travail et d'emploi du personnel infirmier ». On peut les résumer ainsi: 1-Appliquer et étendre, dans le domaine des soins infirmiers, l'étude du travail, la recherche occupationnelle et l'analyse des tâches. $2-\mathrm{La}$ semaine normale moyenne de 40 heures. 3Travail en équipes continues. 4- Vacances annuelles non inférieures à un mois. 5-Rémunération et échelles de traitements calculées sur la base de l'analyse et de l'évaluation des tâches.

\section{Jacpues Archambault}

Manifeste pour une civilisation solidaire, par L.-J. Lebret. Economie et Humanisme, Caluire, 1959, 94 pp.

Tous connaissent l'excellent travail accompli depuis quinze ans par l'équipe «Economie et Humanisme» pour orienter le développement de notre civilisation dans un sens plus humain.

Dans ce Manifeste, le Père Lebret et ses collaborateurs cherchent à alerter sur la situation du monde et à ouvrir une voie qui permettra de sortir des impasses dans lesquelles l'humanité se trouve engagée. «Ce document, diton dans le préambule, voudrait à tout le moins être un cri assez percutant pour percer le mur de la surdité générale, assez angoissé pour répondre à la gravité de la conjoncture, assez humain pour être accueilli avec sympathie, assez constructif pour apporter l'espérance ».

Après avoir expliqué ce qu'il faut entendre par une civilisation solidaire, le Manifeste aborde le problème primordial des pouvoirs sur les biens et de la propriété. Suit ensuite une prise de position devant les forces, idéologies et régimes qui s'affrontent: capitalisme, socialisme, communisme, tiers-monde. Cette critique faite, le Manifeste exprime des positions positives: le recours nécessaire à l'amour fraternel éclairé, l'objectif de «valorisation 》 de l'humanité, l'analyse indispensable des besoins et des possibilités pour orienter les interventions. Dans cette orientation, la coopération doit s'établir avec le mouvement ouvrier, le mouvement rural et les élites authentiques pour préparer les voies à la démocratie et contribuer à l'approche d'une civilisation d'économie humaine et de solidarité universelle.

Ce Manifeste est un appel à tous les hommes de bonne volonté pour essayer de sortir l'humanité des contradictions dans lesquelles elle se débat. «La révolution que nous proposons, y lit-on, est la révolution intensive en profondeur; il s'agit d'une rénovation de l'homme même acquérant la taille qu'il doit obtenir devant une situation du monde si difficile et le contrôle continu de soi dans une société complexe si rapidement évolutive. Il s'agit d'un changement de manière d'être, de manière d'aborder, d'accueillir, de donner et de recevoir; il s'agit d'une reconversion de toutes les structures dans une perspectives d'obiectivité, de complémentarité, de service; il s'agit d'un mouvement universel remuant les consciences, transformant les mentalités et les moeurs, activant les intelligences, provoquant la recherche, mettant au point les méthodes, s'imposant aux dirigeants, dynamisant et disciplinant les masses $\gg$. Cette économie humaine est \&la seule voie possible à l'humanité si elle veut échapper aux barbaries ».

Manifeste pour une économie solidaire est un ouvrage qui doit être lu 